\title{
Validation of refractivity profiles derived from GRAS raw-sampling data
}

\author{
F. Zus ${ }^{1}$, G. Beyerle ${ }^{1}$, S. Heise ${ }^{1}$, T. Schmidt ${ }^{1}$, J. Wickert ${ }^{1}$, and C. Marquardt ${ }^{2}$ \\ ${ }^{1}$ Helmholtz Centre Potsdam, GFZ German Research Centre for Geoscience Potsdam, Germany \\ ${ }^{2}$ EUMETSAT, Darmstadt, Germany
}

Received: 25 February 2011 - Published in Atmos. Meas. Tech. Discuss.: 16 March 2011

Revised: 24 June 2011 - Accepted: 7 July 2011 - Published: 27 July 2011

\begin{abstract}
Results from GRAS (GNSS Receiver for Atmospheric Sounding) RO (Radio Occultation) data recorded in RS (Raw Sampling) mode processed at the GFZ (German Research Centre for Geoscience) Potsdam are presented. The experimental processing software POCS-X includes FSI (Full Spectrum Inversion) in order to cope with multi-path regions and enables in connection with RS data to retrieve atmospheric refractivity profiles down to the Earths surface. Radio occultation events observed between 30 September and 30 October 2007 are processed and the retrievals are validated against co-located ECMWF (European Centre for Medium-Range Weather Forecasts) profiles. The intercomparison indicates good quality of the retrieved profiles. In the altitude range 8 to $25 \mathrm{~km}$ the standard deviation is below $1 \%$. The mean deviation in this altitude range tends to be negative. At $30 \mathrm{~km}$ the negative bias reaches about $-0.4 \%$. Below $8 \mathrm{~km}$ the standard deviation increases, reaching $2.5 \%$ at $2 \mathrm{~km}$. Below $2 \mathrm{~km}$ the mean deviation tends to be negative, reaching $-1.9 \%$ close to the ground. The negative bias mainly stems from the tropical lower troposphere; there, the negative bias reaches $-3 \%$. The tropospheric penetration depth obtained from RS data shows a vast improvement compared to the tropospheric penetration depth typically obtained from CL (Closed Loop) data; $50 \%$ of all retrieved profiles reach $720 \mathrm{~m}$.
\end{abstract}

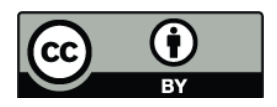

Correspondence to: F. Zus (zusflo@gfz-potsdam.de)

\section{Introduction}

In a RO (Radio Occultation) event, the radio signal transmitted by a GPS (Global Positioning System) satellite, traverses the atmosphere before it is recorded by a receiver aboard a LEO (Low Earth Orbiting) satellite. Refraction in the atmosphere changes the phase and amplitude of the signal. Bending angles are derived and inverted to atmospheric refractivity under the assumption of spherical symmetry. This unique remote sensing method of bending angle/refractivity profiles provides valuable input for numerical weather prediction (Kursinski et al., 1997; Rocken et al., 1997; Wickert et al., 2001, 2009; Anthes et al., 2008; von Engeln et al., 2009; Beyerle et al., 2011).

In the upper troposphere and lower stratosphere good agreement between RO retrievals and meteorological analyses is found. Obtaining accurate retrievals in the lower troposphere (at altitudes below $8 \mathrm{~km}$ ) has proven to be more difficult due to the complicated structure of refractivity there. Advanced receiver tracking methods and the application of sophisticated retrieval algorithms are required. Retrieval algorithms such as the CT (Canonical Transform) (Gorbunov, 2002) or the FSI (Full Spectrum Inversion) (Jensen et al., 2003) allow to determine bending angles in multipath regions. Errors introduced by CL (Closed Loop) tracking are reduced by OL (Open Loop) tracking (Sokolovskiy, 2001). Open Loop tracking is routinely used on the COSMIC (Constellation Observing System for Meteorology, Ionosphere and Climate) mission and the TerraSAR-X mission, and proves to increase the data yield and quality of RO soundings in the lower troposphere (Anthes et al., 2008; Beyerle et al., 2011). Besides improving the ability to probe deeper into the lower troposphere, OL tracking also enables recording rising

Published by Copernicus Publications on behalf of the European Geosciences Union. 
occultations, thus the number of occultation events from the same RO-instrument increases considerably.

The RO-instrument GRAS (GNSS Receiver for Atmospheric Sounding) on-board of EUMETSATs (European Organisation for the Exploitation of Meteorological Satellites) MetOp satellite has been designed for observing setting and rising occultations from the GPS satellite constellation. A dedicated ESA (European Space Agency) funded study was set up to investigate the potential of RO data recorded in RS (Raw Sampling) mode (equivalent to OL tracking). The study exploited a set of in-orbit GRAS data to characterize the performance of the instrument for atmospheric sounding (Bonnedal et al., 2010). A key objective was the analysis of measurement conditions requiring the application of OL tracking and the retrieval of atmospheric profiles. Starting from the same data set, provided by EUMETSAT, participants of the study were asked to retrieve bending angle/refractivity profiles. Among different institutions, i.e. RUAG Space AB, EUMETSAT, DMI (Danish Meteorological Institute), and the University of Graz, the GFZ (German Research Centre for Geoscience) Potsdam participated in this study. A profile-to-profile intercomparsion with other teams in the RS study is ongoing; first results were presented at the OPAC (Occultations for Probing Atmosphere and Climate) workshop in Graz, Austria, 2010 (Marquardt et al., 2010). In this paper we provide a brief description of GFZs in-house experimental processing software package (POCS-X) and compare retrieved refractivity profiles to co-located ECMWF (European Centre for Medium-Range Weather Forecasts) profiles.

This paper is structured as follows. In Sect. 2 we briefly describe the construction of the phase and amplitude from GRAS measurements and the inversion procedure. In Sect. 3 refractivity profiles are compared to ECMWF analyses. Section 4 summarizes the main results.

\section{Processing of GRAS RS data}

In this section we provide a brief description of processing steps leading from RO measurements to refractivity profiles. For further details the reader is referred to e.g. Hajj et al. (2002); Wickert et al. (2004); Sokolovskiy et al. (2009); Beyerle et al. (2011). The processing consists of two stages. In the first processing step, hereinafter referred to as Level 1 processing, L1/L2 atmospheric excess phase paths are derived from the GRAS measurements. In the second processing step, hereinafter referred to as Level 2 processing, excess phase paths are inverted to refractivity profiles.

\subsection{Level 1 processing}

The GRAS instrument normally tracks the $\mathrm{L} 1$ coarse acquisition code (C/A) and the L1/L2 P(Y)-code using a PhaseLocked-Loop (PLL). In this tracking mode (CL mode),
GRAS produces phase and SNR (Signal to Noise Ratio) measurements of the occulting signal at a rate of $50 \mathrm{~Hz}$. The receiver either tracks both the L1 and L2 signals or, at low altitudes $(<5-10 \mathrm{~km})$, the L1 signal is tracked in RS mode parallel to the CL mode. In RS mode, GRAS produces measurements of the occulting signal at a rate of $1 \mathrm{kHz}$ (Bonnedal et al., 2010).

The GRAS instrument outputs NCO (NumericallyControlled Oscillator) phase samples $\phi_{n}^{\text {nco }}$ along with inphase and quadrature-phase correlation sum samples $\hat{I}_{n}=$ $I_{n} / D_{n}$ and $\hat{Q}_{n}=Q_{n} / D_{n}$ respectively. Here, the subscript $n$ denotes sample number, $I_{n}$ and $Q_{n}$ denote the demodulated in-phase and quadrature-phase correlation sum samples and $D_{n}= \pm 1$ denote the navigation data bits. The total phase sample $\phi_{n}$ is reconstructed according to

$\phi_{n}=\phi_{n}^{\mathrm{nco}}+\delta \phi_{n}$

The residual phase sample $\delta \phi_{n}$ is determined through application of the four-quadrant inverse tangent to demodulated in- and quadrature-phase correlation sum samples

$\delta \phi_{n}=\operatorname{atan} 2\left(Q_{n}, I_{n}\right)+c_{n}$

The additional term $c_{n}$ unwraps the residual phase (Beyerle et al., 2006)

$c_{n}=\left\{\begin{array}{l}c_{n-1}+2 \pi: \operatorname{atan} 2\left(Q_{n}, I_{n}\right)-\operatorname{atan} 2\left(Q_{n-1}, I_{n-1}\right)<-\pi \\ c_{n-1}-2 \pi: \operatorname{atan} 2\left(Q_{n}, I_{n}\right)-\operatorname{atan} 2\left(Q_{n-1}, I_{n-1}\right)>+\pi \\ c_{n-1}: \text { otherwise }\end{array}\right.$

with $c_{1}=0$. The residual phase extraction requires demodulated in- and quadrature-phase samples. Thus, knowledge of the navigation data bits is presupposed. The GFZ established a network of ground-based GPS receivers for that purpose (Beyerle et al., 2009). Navigation data bits collected by GFZs ground station network, hereinafter referred to as external navigation data bits, are used whenever available. During the time period considered in this study (September/October in 2007) GFZs ground station network provided navigation data bits with a global coverage of about $75 \%$ (currently the global coverage is about $99 \%$ ). Internal navigation data bits, contained in EUMETSAT NetCDF file, are used if external navigation data bits are not available. Internal navigation data bits are obtained as follows: with the RS tracking at $1 \mathrm{kHz}$ it is possible to find the navigation bits on the carrier phase measurement by analysing 20 sample points (the navigation message is at $50 \mathrm{~Hz}$ ) centred around the known position of the navigation bit. Two phase reconstruction are generated, one as recorded by the receiver, and one with a navigation bit (phase shift) included. An internal navigation bit is identified if the phase reconstruction with phase shift is smoother around the 20 sample points centre position. The reconstruction of the phase following Eqs. (13 ) is done in the same way for CL and RS data.

The phase of the L1 signal is assembled from CL data recorded at $50 \mathrm{~Hz}$ and $\mathrm{RS}$ data recorded at $1 \mathrm{kHz}$. No attempt is made to fill data gaps present in the CL and RS 
data. We select the longest continuous CL record and the longest continuous RS record. In this selection preference is given to $\mathrm{RS}$ data in the $\mathrm{CL} / \mathrm{RS}$ overlap region. If there is a gap between the selected CL and RS record the occultation event is rejected from further processing. If there is no gap between the selected CL and RS record we continue to process the occultation event. The phase of the assembled L1 signal is in general not continuous, i.e. the contiguous CL and RS records deviate by a (constant) phase-offset. This phase-offset is determined from data in the CL/RS overlap region. The resulting continuous L1 signal is downsampled to $50 \mathrm{~Hz}$. The phase of the L2 signal is solely available from $\mathrm{CL}$ data recorded at $50 \mathrm{~Hz}$. The phase data are corrected for relativistic effects and a zero differencing scheme is applied to retrieve L1/L2 atmospheric excess phase paths (Beyerle et al., 2005a).

\subsection{Level 2 processing}

\subsubsection{Standard retrieved bending angles}

For the low-pass filtering of the L1/L2 excess phase paths and the simultaneous calculation of the L1/L2 excess phase path rates (derivative with respect to time) we apply a local polynomial regression of degree 3 using 71 samples (SavitzkyGolay smoothing filter). Assuming a tangent point vertical velocity of $2 \mathrm{~km} \mathrm{~s}^{-1}$ (the sampling rate is $50 \mathrm{~Hz}$ ) a local polynomial regression of degree 3 using 71 samples corresponds to a spatial scale of around $2.2 \mathrm{~km}$. The bending angles $\alpha_{1}$ and $\alpha_{2}$ as functions of the impact parameters $p_{1}$ and $p_{2}$ are calculated from the L1 and L2 excess phase path rates (Kursinski et al., 1997). The bending angle related to the neutral atmosphere is determined through ionospheric calibration using the L1 and L2 bending angles (Hajj et al., 2002). After the low-pass filtering and the ionospheric calibration, statistical optimization is performed to damp bending angle noise (Healy, 2001):

$\alpha=\alpha_{\mathrm{b}}+\frac{\sigma_{\mathrm{b}}^{2}}{\left(\sigma_{\mathrm{b}}^{2}+\sigma_{\mathrm{o}}^{2}\right)}\left(\alpha_{\mathrm{o}}-\alpha_{\mathrm{b}}\right)$

Here $\alpha_{\mathrm{b}}$ and $\alpha_{\mathrm{o}}$ denotes the background and observed bending angle and $\sigma_{\mathrm{b}}$ and $\sigma_{\mathrm{o}}$ denotes the background and observation error variance. The bending angle $\alpha$ represents the optimal estimate taking into account both the background and the observation errors. The statistical optimization according to Eq. (4) does not take into account vertical error correlations, i.e. the backround and observation error covariance matrices are assumed to be diagonal matrices. The background bending angle profile is computed from the co-located MSIS climatology (Hedin, 1991) refractivity profile using the inverse Abel transform (Fjeldbo et al., 1971). The background error variance is taken to be $20 \%$ of the background bending angle (Healy, 2001). The observation error variance is taken to be $1.2 \mu \mathrm{rad}$ (von Engeln et al., 2009). Note that, our statistical optimization is based on a simple approach; we do not differentiate between different occultation events and we do not take into account background error correlations and observation error correlations. For a more sophisticated statistical optimization the reader is referred to e.g. Lauritsen et al. (2011).

\subsubsection{FSI retrieved bending angles}

The arrival times of different frequency components in the RO signal can be determined from the derivatives of the phases of the Fourier Transform of the entire complex RO signal (Jensen et al., 2003). This fact allows for the efficient computation of bending angle profiles within regions of multipath ray propagation. The implementation of the FSI closely follows Beyerle (2005b). The excess phase path related to the neutral atmosphere is determined through ionospheric calibration using the L1 and L2 excess phase paths (Beyerle et al., 2004). Frequency variations caused by radial variations in the radius vectors of the GPS and the LEO satellite are accounted for (Jensen et al., 2003). The RO signal in the time domain is not subject to any filtering. While the bending angle as a function of the impact parameter follows from the derivative of the FSI phase, the valid impact parameter range is determined from the FSI amplitude. Specifically, the bending angle profiles are truncated at that impact parameter value where the smoothed FSI amplitude drops below $50 \%$ of the maximum value. The resolution of the FSI bending angle profile is reduced from $0.5 \mathrm{~m}$ to $50 \mathrm{~m}$ using a running mean.

\subsubsection{Refractivity from RO data}

Since multipath ray propagation is unlikely to occur at high altitudes, FSI retrieved bending angles are replaced by the corresponding standard retrieved bending angles above $12 \mathrm{~km}$ ray-height. Finally, the assembled bending angle profile is inverted to a refractivity profile using the Abel transform (Fjeldbo et al., 1971). The occultation point, the point on Earth's surface to which the retrieved refractivity profile is assigned, is estimated under the tangent point where the GPS-LEO line-of-sight altitude equals $10 \mathrm{~km}$.

\subsection{Refractivity from ECMWF analysis}

ECMWF refractivity is computed from pressure, temperature and water vapor partial pressure (Bevis et al., 1994). Interpolation between grid points $\left(0.5^{\circ} \times 0.5^{\circ}\right.$ horizontal resolution; 91 model levels in the vertical) and linear interpolation in time is performed between $6 \mathrm{~h}$ analyses fields. The discrepancy between the RO refractivity retrieval and the ECMWF refractivity is measured in terms of the fractional refractivity deviation. 


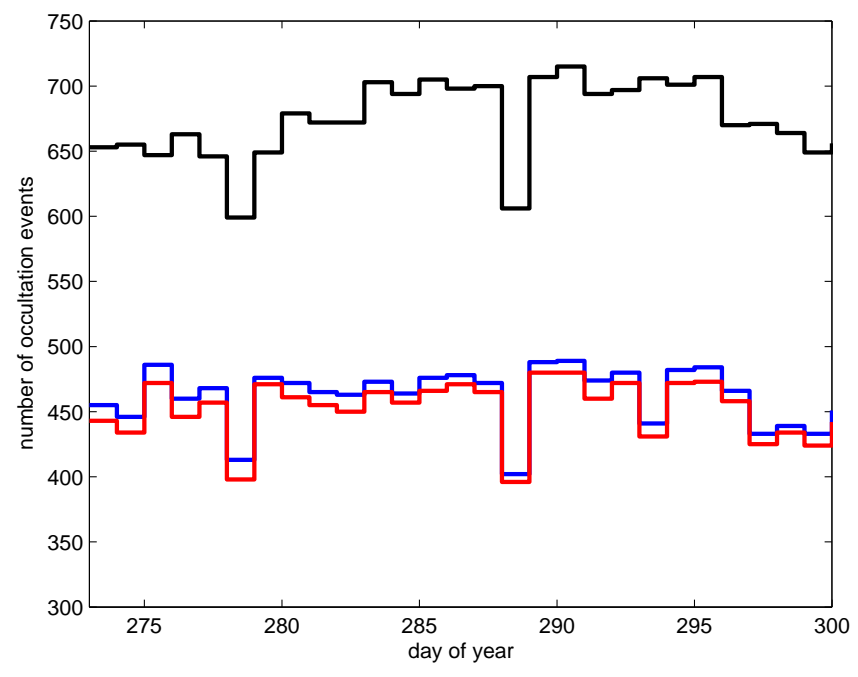

Fig. 1. Number of occultation events versus day of year. Total number of occultations events (black), number of profiles after early stage QC (blue) and number of profiles after final stage QC (red).

\subsection{Quality Control}

Quality Control (QC) is applied at different stages of the processing. The early stage QC is applied at Level 1 and Level 2 and identifies CL and RS data gaps, examines the CL/RS overlap, SNRs, and L1/L2 excess phase path ratios. Specifically, occultation events are rejected if there is a gap between the selected CL and RS record (see Sect. 2.1), the ratio of L1/L2 excess phase path forward differences do not meet the criteria proposed by Beyerle et al. (2004) (excess phase path forward differences are analyzed in the ionospheric calibration procedure) and the retrieved bending angle profile does not cover the altitude range $10-40 \mathrm{~km}$. The final stage QC compares the retrieved refractivity profiles to the ECMWF refractivity profiles. Profiles where the fractional refractivity deviation exceeds $\pm 10 \%$ at any altitude between $5 \mathrm{~km}$ and $30 \mathrm{~km}$ are rejected. No final stage QC is applied for altitudes $<5 \mathrm{~km}$.

\section{Results and discussion}

The focus is on data from September/October 2007 (Day Of Year (DOY): 273-300) provided by EUMETSAT. Out of 18878 occultation events, 12678 occultation events pass our QC. Note that there are significantly more (successfully retrieved) profiles from setting occultation events than rising occultation events. In fact, $60 \%$ of all retrieved profiles stem from setting occultation events. This is due to the fact that in rising occultation events data gaps in the CL portion of the signal are frequent (about $33 \%$ of all rising occultation events are affected). Since our retrieval algorithm relies on the longest continuous CL record and the longest continuous
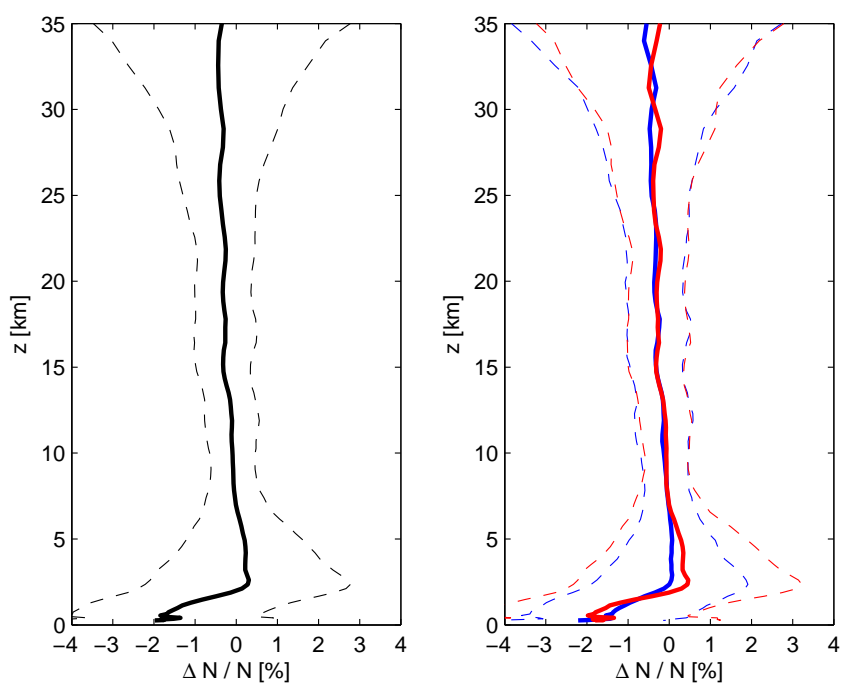

Fig. 2. Left panel: fractional refractivity deviation from the ECMWF analysis for all occultation events versus altitude. Right panel: fractional refractivity deviation from the ECMWF analysis for rising and setting occultation events versus altitude. The blue (red) line coressponds to rising (setting) occultations. The solid line indicates the mean and the dashed lines indicate the \pm one-sigma deviation from the mean.

RS record, and gaps between the selected CL and RS records are not filled (see Sect. 2.1), a large number of rising occultation events are rejected by our QC. Figure 1 shows the total number of occultations, the number of occultations after early stage QC and the number of occultations after final stage QC versus day of year.

\subsection{Upper troposphere and lower stratosphere}

The left panel of Fig. 2 shows the fractional refractivity deviation from the ECMWF analysis (measured in terms of the mean and the standard deviation) versus altitude for all occultation events. The standard deviation between $8 \mathrm{~km}$ to $25 \mathrm{~km}$ is below $1 \%$, confirming the high quality of the GPS RO data in the upper troposphere and lower stratosphere. The mean deviation in this altitude range tends to be negative, reaching $-0.4 \%$ in between 30 and $35 \mathrm{~km}$. This finding is consistent with recent validation studies of operational GRAS RO data (von Engeln et al., 2009). The source of this bias is yet not well understood. The influence of the filter options, the lowpass filter applied to the phase and the statistical optimization applied to the bending angle, can not be ruled out. For example, the role of the low-pass filter was recently discussed in the context of retrievals derived from COSMIC RO data (see the COSMIC page http://cosmic-io.cosmic.ucar.edu/cdaac/ status.html). Likewise, the statistical optimization can introduce a bias, i.e. the background bending angle can be biased. Since we assume that the observation error variance is 

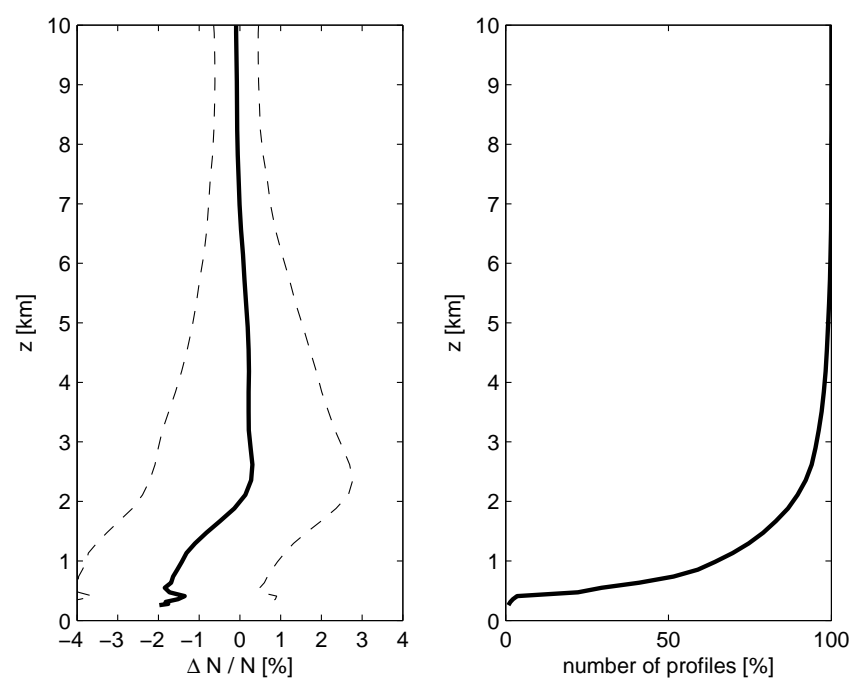

Fig. 3. Fractional refractivity deviation from the ECMWF analysis (left) and number of retrieved data points (right) versus altitude. The solid line indicates the mean, the dashed lines indicate the \pm one-sigma deviation from the mean.

constant, but the background error variance is a fraction of the background bending angle (see Eq. 4), the optimal bending angle automatically tends towards the observed bending angle at low ray-heights. Thus if the negative bias stems from the retrieval, the negative bias is inherent to the observed bending angle. Indeed, the negative bias does not only show up in the fractional deviation of refractivity but also in the fractional deviation of non-optimized bending angles (not shown). However, it is important to note that retrievals derived by different processing centers (different filter options) for different RO missions, e.g. COSMIC and TerraSAR$\mathrm{X}$, show essentially the same negative bias (see the GRAS SAF monitoring page http://www.grassaf.org/monitoring/). Therefore, it can not be excluded that part of the negative bias stems from the ECMWF analysis. The right panel of Fig. 2 shows the fractional refractivity deviation from the ECMWF analysis versus altitude for rising and setting occultation events. While the mean and the standard deviation for rising and setting occultation events are similar above $8 \mathrm{~km}$, below $8 \mathrm{~km}$ we observe distinct differences.

\subsection{Lower troposphere}

At first and for sake of clarity, Fig. 3 shows the fractional refractivity deviation from the ECMWF analysis versus altitude for all occultation events in the lower troposphere. The number of retrieved data points at a given altitude is shown in the right panel of Fig. 3. Below $8 \mathrm{~km}$ the standard deviation is increasing, reaching $2.5 \%$ at $2 \mathrm{~km}$. Below $2 \mathrm{~km}$ the mean deviation tends to be negative, reaching $-1.9 \%$ close to the ground. Though comparatively small, it is worth to
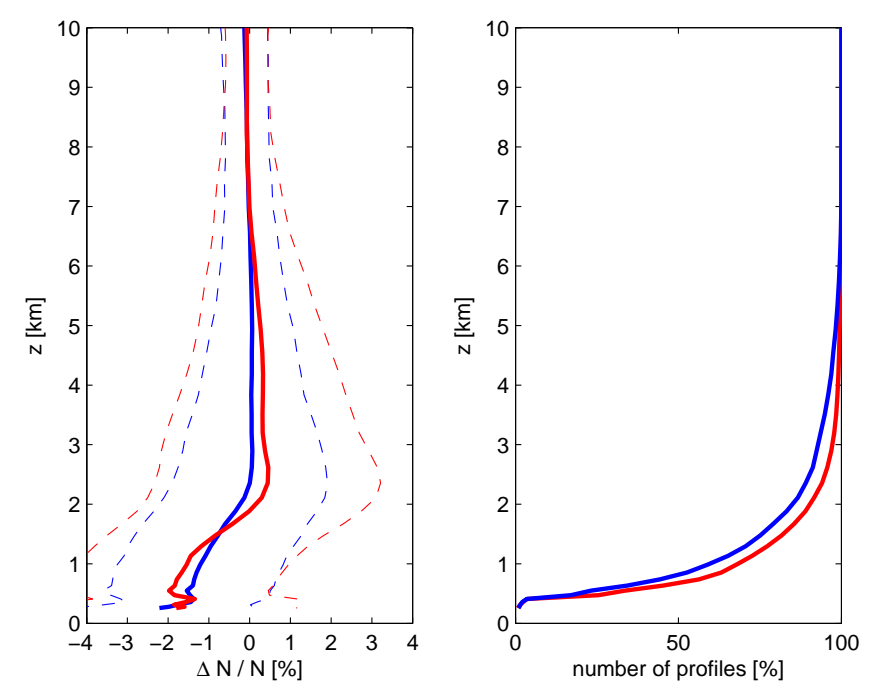

Fig. 4. Fractional refractivity deviation from the ECMWF analysis (left) and number of retrieved data points (right) versus altitude. The blue (red) line coressponds to rising (setting) occultations. The solid line indicates the mean, the dashed lines indicate the \pm one-sigma deviation from the mean.

mention that a small positive bias exists in between 2 and $6 \mathrm{~km}$. The $50 \%$ altitude, defined as the altitude where the number of successfully retrieved data points is reduced to $50 \%$, is $720 \mathrm{~m}$; that is, compared to RO data recorded in CL mode, RO data recorded in RS mode strongly improves the ability to probe deep into the lower troposphere (Gorbunov et al., 2011a). Figure 4 shows the fractional refractivity deviation from the ECMWF analysis versus altitude for rising and setting occultation events in the lower troposphere. The number of retrieved data points at a given altitude is shown in the right panel of Fig. 4. The standard deviation differs by up to $1 \%$ at $2 \mathrm{~km}$. The small positive bias between 2 and $6 \mathrm{~km}$ mentioned previously stems from setting occultations. For both, rising and setting occultations, a pronounced negative bias exists close to the ground. The differences in the fractional refractivity deviation from the ECMWF analysis are accompanied by a difference in the number of retrieved data points at a given altitude; compared to profiles retrieved from rising occultations, profiles retrieved from setting occultations extend deeper into the lower troposphere.

The meridional distribution of the fractional refractivity deviation from the ECMWF analysis at low altitudes shows that the negative and comparatively small positive bias mainly stem from the tropical lower troposphere. It is convenient to separate the occultation events into different latitude bands; the northern hemisphere, ranging from $30^{\circ} \mathrm{N}-90^{\circ} \mathrm{N}$, the southern hemisphere ranging from $30^{\circ} \mathrm{S}-90^{\circ} \mathrm{S}$ and the tropics, ranging from $30^{\circ} \mathrm{S}-30^{\circ} \mathrm{N}$. Figures 5-7 show the fractional refractivity deviation from the ECMWF analysis versus altitude for the northern, southern hemisphere and the 


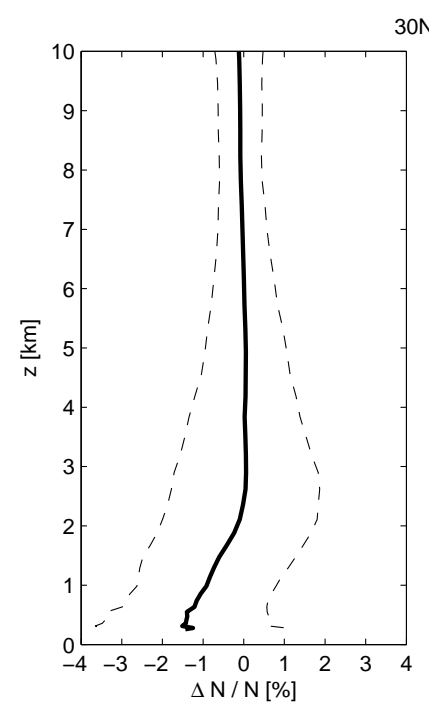

$30 N-90 N$

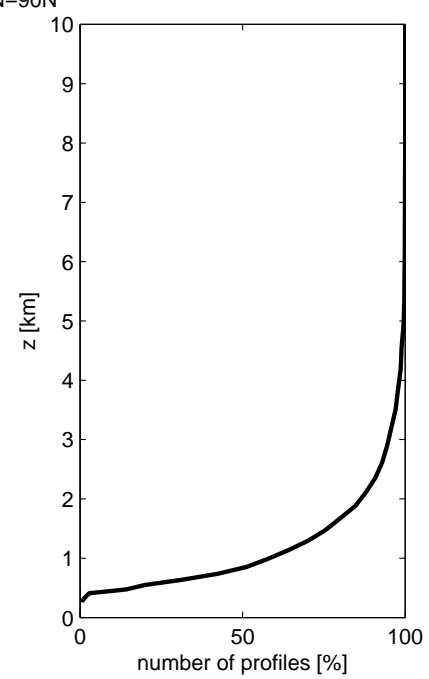

Fig. 5. Fractional refractivity deviation from the ECMWF analysis (left) and number of retrieved data points (right) versus altitude (northern hemisphere: $30^{\circ} \mathrm{N}-90^{\circ} \mathrm{N}$ ). The solid line indicates the mean, the dashed lines indicate the \pm one-sigma deviation from the mean.

tropics. The number of retrieved data points at a given altitude is shown in the right panels of Figs. 5-7. In the northern and southern hemisphere biases are insignificant from $2 \mathrm{~km}$ to $10 \mathrm{~km}$. Below $2 \mathrm{~km}$ the negative bias reaches $-1 \%$ in the northern hemisphere and $-2 \%$ in the southern hemisphere. The standard deviation is well below $2 \%$ for both hemispheres. The $50 \%$ altitude is about $830 \mathrm{~m}$ for northern hemisphere and about $630 \mathrm{~m}$ for the southern hemisphere. In the tropics a pronounced negative bias exists reaching $-3 \%$ close to the ground. The standard deviation reaches $3.5 \%$ at $2 \mathrm{~km}$. The $50 \%$ altitude is about $800 \mathrm{~m}$. For the northern and southern hemisphere we do not find significant differences between rising and setting occultations in terms of the fractional refractivity deviation from the ECMWF analysis and the number of retrieved data points at a given altitude (not shown). Figure 8 shows the fractional refractivity deviation from the ECMWF analysis versus altitude for rising and setting occultation events along with the number of retrieved data points at a given altitude in the tropics. For rising occultations biases are insignificant from $2 \mathrm{~km}$ to $6 \mathrm{~km}$. For setting occultations the bias tends to be positive, reaching $1 \%$ in between 2 and $3 \mathrm{~km}$. The fraction of profiles probing the lower troposphere is significantly larger for setting occultations than for rising occultations.

A possible explanation of the enhanced negative bias below $2 \mathrm{~km}$ is the presence of critical refraction (Ao et al., 2003; Sokolovskiy, 2003); in particular in the tropics a frequently observed phenomenon. For example, Beyerle et al. (2006) estimated that in the tropics about $58 \%$ of all occultation

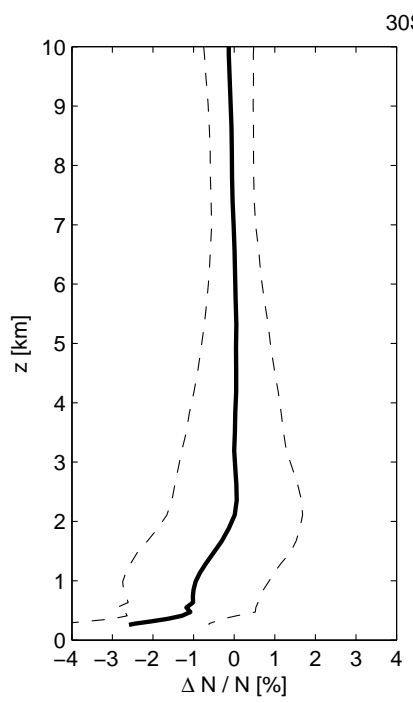

$30 S-90 S$

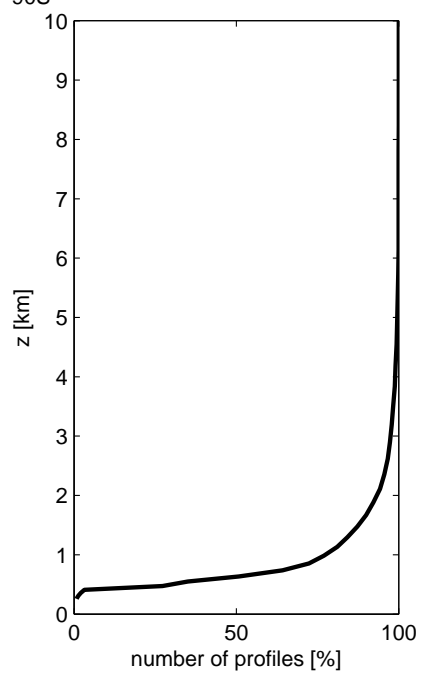

Fig. 6. Fractional refractivity deviation from the ECMWF analysis (left) and number of retrieved data points (right) versus altitude (southern hemisphere: $30^{\circ} \mathrm{S}-90^{\circ} \mathrm{S}$ ). The solid line indicates the mean, the dashed lines indicate the \pm one-sigma deviation from the mean.

events are affected by critical refraction. End-to-end simulations performed in the same study (including a receiver operating in OL mode implemented in software) suggest that critical refraction introduces a mean error of about $-1 \%$; the standard deviation is estimated to be about $2 \%$. Another possible source of the negative bias in the lower troposphere are strong horizontal gradients as discussed by Gorbunov et al. (2010). In addition recent investigations by Sokolovskiy et al. (2010) point to the fact that the truncation and filtering of RO signals have a large influence on both, positive and negative, biases in the tropical lower troposphere. In particular, these investigations indicate that the truncation of RO signals (in the time domain) tends to enhance the negative bias and simultaneously reduce the positive bias (mean deviation between COSMIC and ECMWF). Figure 9 shows the frequency distribution (normed to unity) of the minimum SLTA (Straight Line Tangent point Altitude) for rising and setting occultation events in the tropics for October 2007, DOY: 280-286. The SLTA corresponds to the altitude of the tangent point above Earth's surface of the straight line between the GPS and LEO satellite. The mean minimum SLTA for setting occultations is $-128 \mathrm{~km}$. The mean minimum SLTA for rising occultations is $-116 \mathrm{~km}$. The question arises to what extent (if at all) this mean minimum SLTAs affects the negative and positive bias in the tropical lower troposphere. This question can only be answered by part: we can not decrease the minimum SLTA (we do not have data below the minimum SLTA) but only increase the minimum SLTA. Here we use some ad hoc procedure to truncate the RO signal: the noise level of the RO signal is estimated by 


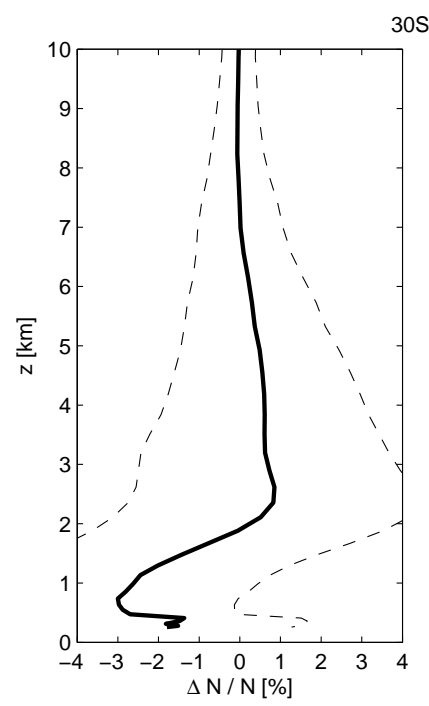

$30 \mathrm{~S}-30 \mathrm{~N}$

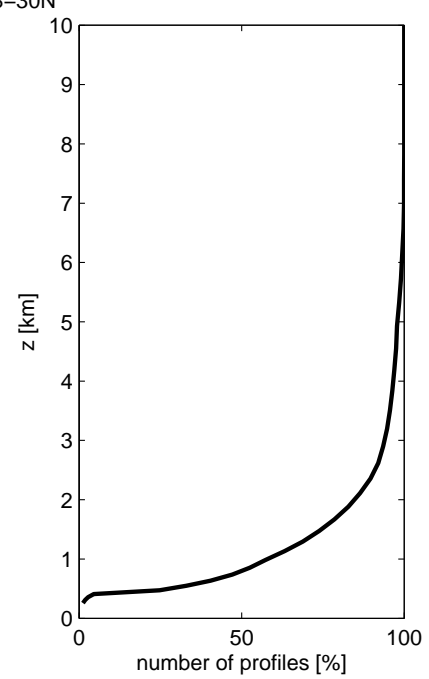

Fig. 7. Fractional refractivity deviation from the ECMWF analysis (left) and number of retrieved data points (right) versus altitude (tropics: $30^{\circ} \mathrm{S}-30^{\circ} \mathrm{N}$ ). The solid line indicates the mean, the dashed lines indicate the \pm one-sigma deviation from the mean.

averaging the amplitude over the last $3 \mathrm{~s}$ at the bottom of an occultation. The RO signal is used for inversion above lowest SLTA where the amplitude, smoothed with a $1 \mathrm{~s}$ window, exceeds the noise level by $50 \%$. We restrict this procedure to RO signals with a minimum SLTA $<-100 \mathrm{~km}$. This procedure is similar to the one proposed by Sokolovskiy et al. (2009). Figure 10 shows the corresponding frequency distribution (normed to unity) of the minimum SLTA for rising and setting occultation events in the tropics. The mean minimum SLTA for setting occultations is now $-112 \mathrm{~km}$. The mean minimum SLTA for rising occultations is now $-100 \mathrm{~km}$. Figures 11 and 12 show the fractional refractivity deviation from the ECMWF analysis versus altitude for rising and setting occultations respectively. Again, the number of retrieved data points at a given altitude is shown in the right panel of Figs. 11 and 12. Indeed, we find that if the RO signal is truncated the negative bias is enhanced while the positive bias is decreased. The exact magnitude of biases in the lower tropical troposphere is at least to some extent affected by the length of the RO signal used in the inversion procedure. Since we truncate the RO signal in the time domain, but we do not alter the truncation procedure in the impact parameter domain, the fraction of profiles probing the lower troposphere is reduced accordingly.
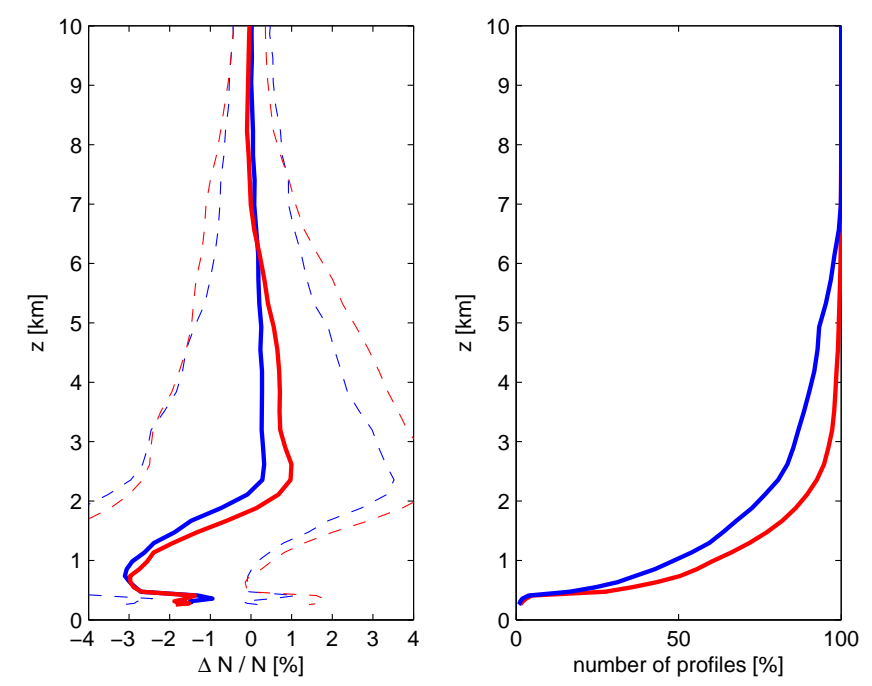

Fig. 8. Fractional refractivity deviation from the ECMWF analysis (left) and number of retrieved data points (right) versus altitude (tropics: $30^{\circ} \mathrm{S}-30^{\circ} \mathrm{N}$ ). The blue (red) line corresponds to rising (setting) occultations. The solid line indicates the mean, the dashed lines indicate the \pm one-sigma deviation from the mean.

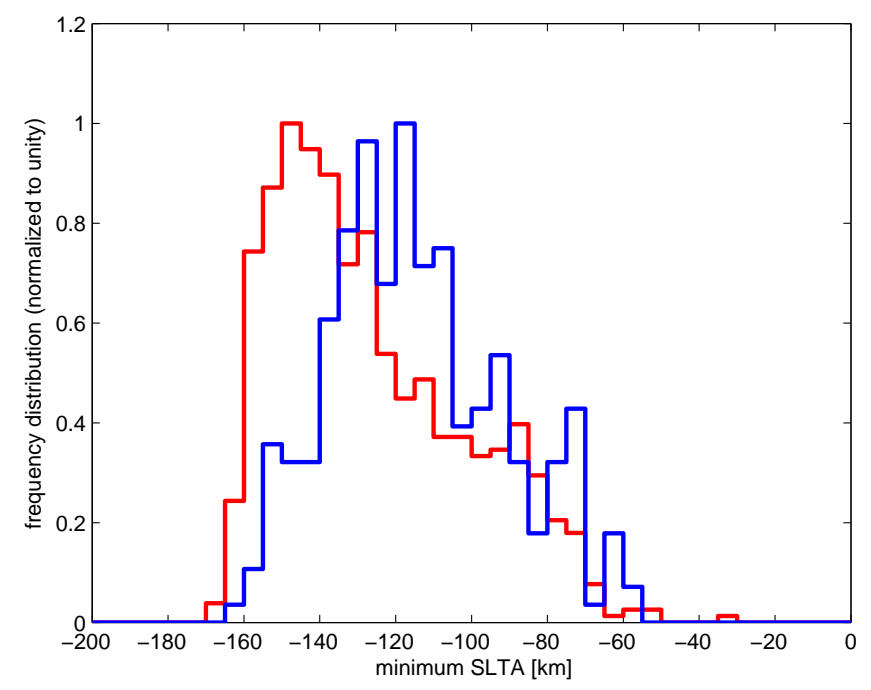

Fig. 9. Normalized frequency distribution of the minimum SLTA. The blue (red) line corresponds to rising (setting) occultations. The data is taken from October 2007, DOY: 280-286.

\section{Conclusions}

Preliminary validation efforts of refractivity profiles derived from GRAS RS data indicate good quality when compared to refractivity profiles derived from the ECMWF analysis. In the altitude range 8 to $25 \mathrm{~km}$ the standard deviation is below $1 \%$. The mean deviation in this altitude range tends to be negative. At $30 \mathrm{~km}$ the negative bias reaches about 


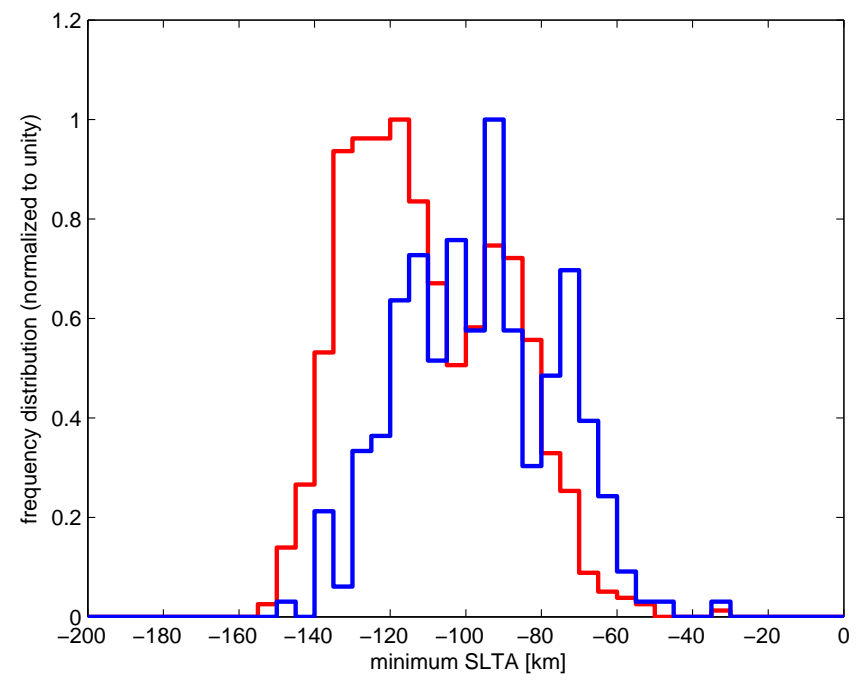

Fig. 10. Same as Fig. 9. The RO signal is truncated (for details refer to Sect. 3.2).
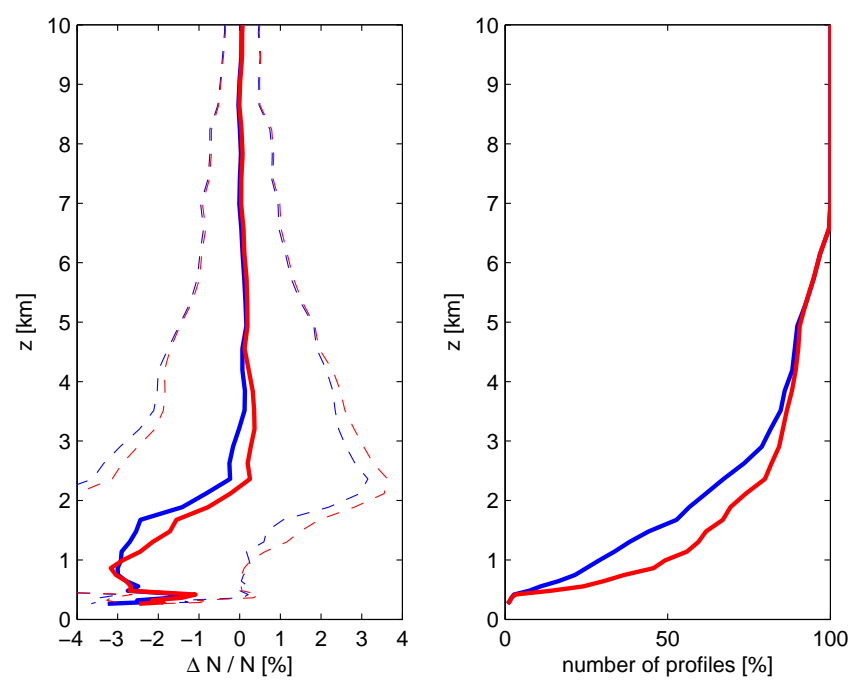

Fig. 11. Fractional refractivity deviation from the ECMWF analysis (left) and number of retrieved data points (right) versus altitude (tropics: $30^{\circ} \mathrm{S}-30^{\circ} \mathrm{N}$ ) for rising occultations. The blue (red) line corresponds to the case when the RO signal is (is not) truncated. For details refer to Sec. 3.2. The solid line indicates the mean, the dashed lines indicate the \pm one-sigma deviation from the mean. The intercomparison is restricted to data from October 2007, DOY: 280286.

$-0.4 \%$. This feature is also observed by other RO missions, e.g. COSMIC and TerraSAR-X.

Raw sampling and the application of an advanced retrieval algorithm work as intended, allowing for refractivity profiles that extend deep into the lower troposphere. The $50 \%$ altitude is $720 \mathrm{~m}$, a vast improvement compared to a $50 \%$ alti-
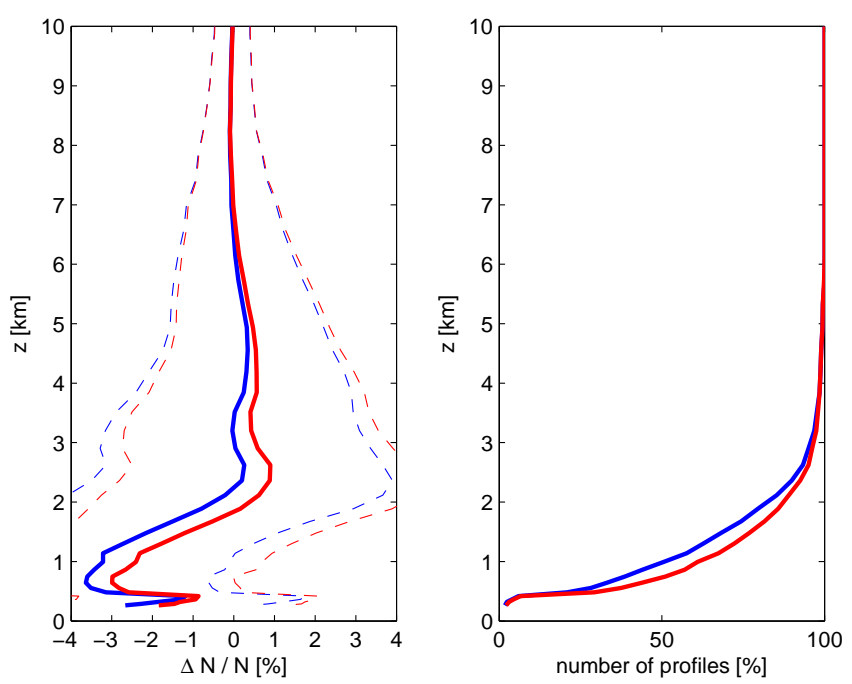

Fig. 12. Fractional refractivity deviation from the ECMWF analysis (left) and number of retrieved data points (right) versus altitude (tropics: $30^{\circ} \mathrm{S}-30^{\circ} \mathrm{N}$ ) for setting occultations. The blue (red) line corresponds to the case when the RO signal is (is not) truncated. For details refer to Sect. 3.2. The solid line indicates the mean, the dashed lines indicate the \pm one-sigma deviation from the mean. The intercomparison is restricted to data from October 2007, DOY: 280 286.

tude of about $3 \mathrm{~km}$ typically obtained from CL data. In the tropical lower troposphere a pronounced negative bias exists, reaching $-3 \%$. A small positive bias exists, reaching about $1 \%$ in between 2 and $3 \mathrm{~km}$. The exact magnitudes of these biases depend at least to some extent on the length of the RO signal used in the inversion procedure, i.e. the truncation of RO signals tends to reduce the positive bias and enhances the negative bias.

Currently $68 \%$ of all occultations pass our QC, corresponding to about 450 profiles per day. An increased yield of the retrievals, i.e. a method to fill data gaps in the CL portion of the RO signal, is work in progress. The profile-to-profile intercomparison with other teams participating in the GRAS RS study is on-going. Cross-center comparison studies (Marquardt et al., 2010; Gorbunov et al., 2011b; Zus et al., 2011) will be beneficial to study uncertainties in different processing software packages.

Acknowledgements. The European Centre of Medium-Range Weather Forecasts provided meteorological analysis fields. The RS study was funded by the European Space Agency under contract 21995/08/NL/EL.

Edited by: K. B. Lauritsen 


\section{References}

Anthes, R. A., Bernhardt, P. A., Chen, Y., Cucurull, L., Dymond, K. F., Ector, D., Healy, S. B., Ho, S.-P., Hunt, D. C., Kuo, Y.-H., Liu, H., Manning, K., McCormick, C., Meehan, T. K., Randel, W. J., Rocken, C., Schreiner, W. S., Sokolovskiy, S. V., Syndergaard, S., Thompson, D. C., Trenberth, K. E., Wee, T.-K., Yen, N. L., and Zeng, Z.: The COSMIC/FORMOSAT-3 Mission: Early Results, B. Am. Meteorol. Soc., 83, 313-333, doi:10.1175/BAMS89-3-313, 2008.

Ao, C. O., Meehan, T. K., Hajj, G. A., Mannucci, A. J., and Beyerle, G.: Lower-troposphere refractivity bias in GPS occultation retrievals, J. Geophys. Res., 108(D18), 4577, doi:10.1029/2002JD003216, 2003.

Bevis, M., Businger, S., Chiswell, S., Herring, T. A., Anthes, R. A., Rocken, C., and Ware, R. H.: GPS meteorology: Mapping zenith wet delays onto precipitable water, J. Appl. Meteorol., 33(3), 379-386, 1994.

Beyerle, G., Wickert, J., Schmidt, T., and Reigber, C.: Atmospheric sounding by global navigation satellite system radio occultation: An analysis of the negative refractive bias using CHAMP observations, J. Geophys. Res., 109, D01106, doi:10.1029/2003JD003922, 2004.

Beyerle, G., Wickert, J., Schmidt, T., and Reigber, C.: GPS radio occultation with GRACE: Atmospheric profiling utilizing the zero difference technique, Geophys. Res. Lett., 32, L13806, doi:10.1029/2005GL023109, 2005a.

Beyerle, G.: Simulating GPS radio occultation events, Scientific report, 05-09, Danish Meteorological Institute (DMI), 1-38, 2005b.

Beyerle, G., Schmidt, T., Wickert, J., Heise, S., Rothacher, M., König-Langlo, G., and Lauritsen, K. B.: Observations and simulations of receiver-induced refractivity biases in GPS radio occultation, J. Geophys. Res., 111, D12101, doi:10.1029/2005JD006673, 2006.

Beyerle, G., Ramatschi, M., Galas, R., Schmidt, T., Wickert, J., and Rothacher, M.: A data archive of GPS navigation messages, GPS Solutions, 13, 35-41, doi:10.1007/s10291-008-0095-y, 2009.

Beyerle, G., Grunwaldt, L., Heise, S., Köhler, W., König, R., Michalak, G., Rothacher, M., Schmidt, T., Wickert, J., Tapley, B. D., and Giesinger, B.: First results from the GPS atmosphere sounding experiment TOR aboard the TerraSAR-X satellite, Atmos. Chem. Phys., 11, 6687-6699, doi:10.5194/acp-11-66872011, 2011.

Bonnedal, M., Christensen, J., Carlström, A., and Berg, A.: MetopGRAS in-orbit instrument performance, GPS Solutions, 14, 109120, doi:10.1007/s10291-009-0142-3, 2010.

Fjeldbo, G., Kliore, A. J., and Eshleman, V. R.: The neutral atmosphere of Venus as studied with the Mariner V radio occultation experiments, Astron. J., 76(2), 123-140, 1971.

Gorbunov, M. E.: Canonical transform method for processing radio occultation data in the lower troposphere, Radio Sci., 37(5), 1076, doi:10.1029/2000RS002592, 2002.

Gorbunov, M. E., Lauritsen, K. B., and Leroy, S. S.: Application of Wigner distribution function for analysis of radio occultations, Radio Sci., 45, 6011, doi:10.1029/2010RS004388, 2010.

Gorbunov, M. E., Lauritsen, K. B., Benzon, H.-H., Larsen, G. B., Syndergaard, S., and Sørensen, M. B.: Processing of GRAS/METOP radio occultation data recorded in closed-loop and raw-sampling modes, Atmos. Meas. Tech., 4, 1021-1026, doi:10.5194/amt-4-1021-2011, 2011a.

Gorbunov, M. E., Shmakov, A. V., Leroy, S. S., and Lauritsen, K. B.: COSMIC radio occultation processing: Cross-center comparison and validation, J. Atmos. Oceanic Tech., 28, 737-751, doi:10.1175/2011JTECHA1489.1, 2011b.

Hajj, G. A., Kursinski, E. R., Romans, L. J., Bertiger, W. I., and Leroy, S. S.: A technical description of atmospheric sounding by GPS occultation, J. Atmos. Solar-Terr. Phys., 64, 451-469, 2002.

Hedin, A. E.: Extension of the MSIS thermosphere model into the middle and lower atmosphere, J. Geophys. Res., 96, 1159$1172,1991$.

Healy, S. B.: Smoothing radio occultation bending angles above 40 km, Ann. Geophys., 19, 459-468, doi:10.5194/angeo-19-4592001, 2001.

Jensen, A. S., Lohmann, M., Benzon, H.-H., and Nielsen, A.: Full spectrum inversion of radio occultation signals, Radio Sci., 38(3), 1040, doi:10.1029/2002RS002763, 2003.

Kursinski, E. R., Hajj, G. A., Schofield, J. T., Linfield, R. P., and Hardy, K. R.: Observing Earths atmosphere with radio occultation measurements using Global Positioning System, J. Geophys. Res., 19, 23429-23465, doi:10.1029/97JD01569, 1997.

Lauritsen, K. B., Syndergaard, S., Gleisner, H., Gorbunov, M. E., Rubek, F., Sørensen, M. B., and Wilhelmsen, H.: Processing and validation of refractivity from GRAS radio occultation data, Atmos. Meas. Tech. Discuss., 4, 2189-2205, doi:10.5194/amtd-42189-2011, 2011.

Marquardt, C. and the GRAS Raw Sampling study team: Overview on GRAS Raw Sampling and Data Quality Aspects, presentation at the OPAC-4 workshop, Graz, Austria, 2010.

Rocken, C., Anthes, R., Exner, M., Hunt, D., Sokolovskiy, S., Ware, R., Gorbunov, M., Schreiner, W., Feng, D., Herman, B., Kuo, Y.-H., and Zou, X.: Analysis and validation of GPS/MET data in the neutral atmosphere, J. Geophys. Res., 102, 29849-29866, doi:10.1029/97JD02400, 1997.

Sokolovskiy, S.: Tracking tropospheric radio occultation signals from low Earth orbit, Radio Sci., 36(3), 483-498, 2001.

Sokolovskiy, S.: Effect of superrefraction on inversions of radio occultation signals in the lower troposphere, Radio Sci., 38(3), 1058, doi:10.1029/2002RS002728, 2003.

Sokolovskiy, S., Rocken, C., Schreiner, W., Hunt, D., and Johnson, J.: Postprocessing of L1 GPS radio occultation signals recorded in open-loop mode, Radio Sci., 44, RS2002, doi:10.1029/2008RS003907, 2009.

Sokolovskiy, S., Rocken, C., Schreiner, W., and Hunt, D.: On the uncertainty of radio occultation inversions in the lower troposphere, J. Geophys. Res., 115, D22111, doi:10.1029/2010JD014058, 2010.

von Engeln, A., Healy, S., Marquardt, C., Andres, Y., and Sancho, F.: Validation of operational GRAS radio occultation data, Geophys. Res. Lett., 36, L17809, doi:10.1029/2009GL039968, 2009.

Wickert, J., Reigber, C., Beyerle, G., König, R., Marquardt, C., Schmidt, T., Grunwaldt, L., Galas, R., Meehan, T., Melbourne, W. G., and Hocke, K.: Atmosphere sounding by GPS radio occultation: First Results from CHAMP, Geophys. Res. Lett., 28(17), 3263-3266, 2001.

Wickert, J., Schmidt, T., Beyerle, G., König, R., Reigber, C., and Jakowski, N.: The radio occultation experiment aboard CHAMP: Operational data processing and validation of atmospheric parameters, J. Meteorol. Soc. Jpn., 82, 381-395, 2004. 
Wickert, J., Michalak, G., Schmidt, T., Beyerle, G., Cheng, C., Healy, S., Heise, S., Huang, C., Jakowski, N., Köhler, W., Mayer, C., Offiler, D., Ozawa, E., Pavelyev, A., Rothacher, M., Tapley, B., and Arras, C.: GPS radio occultation: Results from CHAMP, GRACE and FORMOSAT-3/COSMIC, Terrestrial, Atmos. Ocean. Sci., 20, 35-50, doi:10.3319/TAO.2007.12.26.01(F3C), 2009.
Zus, F., Beyerle, G., Heise, S., Schmidt, T., and Wickert, J.: Validation of refractivity profiles derived from MetOp and TerraSAR$\mathrm{X}$ open-loop data, presentation at the Fifth FORMOSAT3/COSMIC Data Users Workshop, Taipei, Taiwan, 2011. 